\title{
Teaming up census and patient data to delineate fine-scale hospital service areas and identify geographic disparities in hospital accessibility
}

\author{
Peng Jia $(\mathbb{D}) \cdot$ Xinyu Shi $\cdot$ Imam M. Xierali
}

Received: 2 March 2017 / Accepted: 20 March 2019

(C) The Author(s) 2019

\begin{abstract}
The number of hospital beds per capita, an important measure of equity in healthcare availability and resource allocation, was found to vary across geographic areas in many countries, including the USA. The hospital
\end{abstract}

Peng Jia and Xinyu Shi contributed equally to this work.

This article is part of the Topical Collection on Geospatial Technology in Environmental Health Applications.

Electronic supplementary material The online version of this article (https://doi.org/10.1007/s10661-019-7413-4) contains supplementary material, which is available to authorized users.

\section{P. Jia}

Faculty of Geo-Information Science and Earth Observation (ITC), University of Twente, 7500 Enschede, The Netherlands

\section{P. Jia $(\bowtie) \cdot X$. Shi}

International Initiative on Spatial Lifecourse Epidemiology (ISLE), 7500 Enschede, The Netherlands

e-mail: p.jia@utwente.nl

\section{Shi}

University College Twente, University of Twente, 7500 Enschede, The Netherlands

X. Shi

Department of Educational Leadership and Policy, Graduate School of Education, University at Buffalo, The State University of New York, New York, NY 14260, USA

I. M. Xierali

Department of Family and Community Medicine, University of Texas Southwestern Medical Center, Dallas, TX 75390, USA

I. M. Xierali

Department of Geography and Anthropology, Louisiana State University, Baton Rouge, LA 70803, USA service areas (HSAs) have proven to be more meaningful spatial units for studying health-seeking behaviors and health resource allocation and service utilization. However, when evaluating the geographical balance in ratios of hospital beds to population (HBtP), no existing HSA delineation methods directly consider the underlying population distribution. Using Geographic Information Systems (GIS), this study incorporated the State Inpatient Database with census data to develop a population-based HSA delineation method. The census-derived HSAs were produced for Florida and were validated by aggregating and comparing with the traditional flow-based HSAs. The difference in current ratios of HBtP between the most over- and under-served HSAs was approximately 60 times. Significant clusters of high and low ratios were found in Miami and Jacksonville metropolitan areas, respectively. Such results may be of interest to relevant stakeholders and contribute to planning and optimization of hospital resource allocation and healthcare policy-making. Furthermore, the discovery of a strong correlation between the numbers of hospital discharges and the population at ZIP code level holds a remarkable potential for affordable population estimation, especially in non-census years.

Keywords Accessibility · Census · Florida · GIS . HCUP. Hospital discharge · Hospital service area · Regionalization

\section{Introduction}

The number of hospital beds per capita is an important measure of healthcare resource allocation and equity in 
healthcare availability and accessibility (Anderson et al. 2005; Jia et al. 2015, 2017a; Krueger et al. 2009; Mansfield et al. 1999). Insufficient hospital beds cannot meet the demands of patients in local areas. Wherever healthcare resources are scarce, patients either incur long waits to be seen in local hospitals or travel to distant ones for care (Jackson et al. 2002). Under these circumstances, patients may be prevented from receiving timely care, and the patient-healthcare provider relationships can deteriorate (Hogan 1988; Jones et al. 2008; Moist et al. 2008; Wilbush 1974). Hospital beds per capita have been found to vary across geographic areas in many countries, including the USA, where the number of hospital beds per 1000 population was reported to be 2.9 on average in 2011, according to the World Bank collection of development indicators (Horev et al. 2004; Rodwin and Sandier 1993; Smith 1998; The World Bank 2017).

To mitigate the risk of negative impacts from scarce healthcare resources and optimize the current hospital resource allocation, the HBtP ratios need to be balanced across regions; this will also lead to adequate hospital capacity planning and accurate assessment of real-world healthcare utilization (Ashton et al. 1999; Fisher et al. 1994; Wennberg 1999). A basic solution is regionalization (Claval 1987), which divides an area (e.g., county, state/province, or country) into mutually exclusive spatial units with proper sizes and, based on this, evaluates the per capita share of healthcare resources. Nevertheless, it is argued that existing administrative boundaries are not aligned with the underlying travel behaviors of patients for healthcare purposes and therefore lack the functional healthcare applications presented by hospital service areas (HSAs) (Ashton et al. 1999; Jia et al. 2017a; Klauss et al. 2005; Zhang et al. 2012). An HSA unit is a relatively self-contained healthcare service area within which most of local hospitalization occurs (Center for Evaluative Clinical Sciences 1999). An overarching goal of defining HSAs is to create equitable care across these meaningful units (Jia et al. 2015), ultimately identifying and eliminating inequalities in hospital resource allocation and other factors associated with distant hospitalization (Jia and Xierali 2015).

Despite the availability of spatially finer-scale and socio-demographically homogeneous census units, e.g., census tract (CT), block group (BG), and block, HSAs resulting from previous delineation approaches are mainly aggregated by ZIP codes which are coarser than census units (Center for Evaluative Clinical Sciences
1999; Jia et al. 2015; Klauss et al. 2005). Such compromise is reasonable and accepted in most cases; after all, ZIP code is the finest geographic unit at which level patients' locations of residence are reported in publicly available data (Agency for Healthcare Research and Quality 2011; Jia et al. 2017a). However, previous research suggests that substituting CTs for ZIP codes could significantly increase the accuracy of estimating future demand (Miller 1994). Furthermore, the importance of the population information within functional units has been stated for the success of the task of calculating hospital capacity (Patel et al. 2008). Lack of consideration of the population information in HSA delineation methodology may lead to too small or too large population within HSAs and, therefore, a biased relationship between supply and demand (Shortt et al. 2005). This study filled this gap by aggregating the spatial units reflecting underlying population distribution into HSAs, which would remarkably improve the usefulness of the derived HSAs, especially for such purposes as assessing per capita share of resources.

Against this background, this pilot study aimed to (1) integrate census data with hospital patient data to develop an approach for producing a set of population-based HSAs and (2) demonstrate the spatial disparities in current ratios of HBtP at the HSA level. This easy-toimplement approach bridges geographic and healthcare fields, which particularly serves healthcare planners for scrutinizing geographic variation in per capita share of healthcare resources on a finer geographic scale and for producing regionally and internationally comparable findings relative to the extent of equity in the distribution of healthcare resources.

\section{Methods}

Study area and data

Florida, consisting of 67 counties, is situated in the southeastern USA with three facets bordered by water: the Gulf of Mexico to the west, the Florida Straits between the USA and Cuba to the south, and the North Atlantic Ocean to the east. Therefore, compared to other individual states of the USA, the edge effect in terms of the tendency of patients to travel across state boundaries for hospital care is assumed to be minimized, which makes Florida an ideal study area for investigating patients' healthcare travel patterns in one state. According 
to the 2010 Census (US Census Bureau 2014), a total population of roughly 18.8 million was registered in Florida, with a median age of 40.7 years and the percentages of age groups $<18,18-44,45-64$, and $\geq$ 65 years being $21.3 \%, 34.4 \%, 27.0 \%$, and $17.3 \%$, respectively. The reference day used for the census was April 1, 2010 (National Census Day).

The State Inpatient Database (SID) has been assembled, edited, and standardized by the Agency for Healthcare Research and Quality (AHRQ) as part of the Healthcare Cost and Utilization Project (HCUP) (Agency for Healthcare Research and Quality 2011). Developed through a federal-state-industry partnership, the AHRQ disseminated the HCUP data to provide a large-scale resource of national, state, and allpayer healthcare data and to enhance nationwide comparability among independent health outcomes in different states. This study was based on a set of 2,376,743 inpatient discharge records in 2011 from all 221 general hospitals in Florida, as reported in the 2011 Florida SID data. The 2010 Florida ZIP code boundaries were used to match patients' locations of residence in SID data. Thus, individual records were aggregated into the volume of discharges from each hospital to each ZIP code or, conversely, considered as patient-to-hospital travel flows.

The 2010 Census with numbers of total population within three-tier hierarchical census units in Florida includes 4245 CTs, 11,442 BGs, and 484,481 blocks. Previous studies have aggregated 983 ZIP codes into HSAs in Florida (Center for Evaluative Clinical Sciences 1999; Jia et al. 2017a, b). The BGs with the numbers of total population were chosen as the finest spatial units in this pilot study and considered as a good balance of being fine-scaled (relative to CTs) and not being overly and unnecessarily fine-scaled (disaggregating patients to blocks would introduce more uncertainties due to unknown locations of residence of patients).

The number of hospital beds in 2011 was derived from the 2013 American Hospital Association's (AHA) survey files, ranging from 15 to 2170 with a mean of 252 and a median of 195 . The primary and secondary road networks were available in Geographic Information Systems (GIS) format, published in 2014 by the Florida Department of Transportation. Prior to network analysis, preprocessing was conducted in the ArcGIS editing environment (Version 10.4.1, ESRI, Redlands, CA) to ensure a fully connected road network. As a baseline for comparison, the Dartmouth-derived HSAs in Florida, produced by a traditional flow-based method (Appendix 1), were obtained from Jia et al. (2015).

Matching census and patient data

To assign each BG to the hospital discharging most patients in that BG (i.e., aggregating BGs into HSAs), the numbers of discharges within BGs are needed to be estimated. This necessitated building a relationship between the numbers of discharges and the population. The locational information of the patients was only available at ZIP code level. However, the population numbers within ZIP codes were not directly available from the census data and BG boundaries were not aligned with ZIP code boundaries (BGs were nested within CTs instead of ZIP codes). Therefore, an areal weighting interpolator (AWI) method (Goodchild and Lam 1980) was used to interpolate the population within ZIP codes from populated BGs. Implementation began with intersecting the ZIP code layer and the BG layer. Some BGs were completely located within ZIP codes, but some were divided into two or more intersected zones by ZIP code boundaries. The AWI method assumes that population is uniformly distributed within a BG. Thus, the population in each BG, if divided, was apportioned to each intersected zone on a basis of the areal proportion of that intersected zone over the BG. The estimated population in all intersected zones and BGs completely located within each ZIP code was then added to yield the total population in that ZIP code. As a result, a high correlation (Pearson's $r=0.92$ ) was found between the numbers of hospital discharges and the population at ZIP code level. Hence, a reasonable assumption was made that the percentage of discharges in a BG over a ZIP code approximated to the percentage of the population in that BG over that ZIP code.

For the sake of simplicity, BGs and the intersected zones by BGs and ZIP codes were both referred to as BGs without differentiation for the remainder of this study.

Delineating census-derived HSAs

Before assigning each $\mathrm{BG}$ to the hospital discharging most of its patients, it is worth recalling that the traditional approaches of HSA delineation all assign a ZIP code to the hospital that either actually (Center for Evaluative Clinical Sciences 1999; Klauss et al. 2005) or potentially discharges the largest number of patients in that ZIP code (Jia et al. 2017b). An important 
motivation to replace ZIP codes with BGs as basic units, in addition to availability of the population numbers at BG level, is to better handle some uncertain cases where comparable percentages (e.g., $51 \%$ and $49 \%$ ) of discharges in a ZIP code were from different hospitals at completely different locations, but that ZIP code was only assigned to one hospital (i.e., one that discharged $51 \%$ of patients). With that been said, those ZIP codes with a relatively large percentage of discharges from one hospital can still be reasonably assigned to that hospital without needing to be disassembled into BGs. Therefore, the percentage of discharges from each hospital to each ZIP code was computed based on all patient-tohospital travel flows, and each ZIP code with the highest percentage of discharges (from hospital A) equal to or greater than two times the second highest percentage of discharges (from hospital B) was assigned to hospital A. Each continuous cluster of ZIP codes assigned to the same hospital, with that hospital located within the cluster, formed an initial HSA.

Next, with each initial HSA substituting for the hospital(s) situated within it, patient-to-hospital flows were re-constructed and the percentage of discharges from each hospital/HSA to each unassigned ZIP code was re-computed. The hospital/ HSA discharging the highest percentage of patients to a given ZIP code was assigned as the first hospital/HSA to that ZIP code. If that percentage was smaller than two times the next highest percentage of discharges from a different hospital/HSA, then this hospital/HSA was assigned as the second hospital/HSA to that ZIP code; otherwise, if that percentage was equal to or greater than two times the next highest percentage, or the next highest percentage was below $10 \%$, then assigning ended. This procedure was repeated for each ZIP code until either assigning ended or the fifth hospital was assigned (Fig. 1). As a result, each ZIP code was assigned a varied number of hospitals/HSAs between one and five, with corresponding percentage(s) of discharges all equal to or greater than $10 \%$.

To assign the unassigned ZIP codes which had been split up into BGs (and intersected zones) after the intersection process to their first-fifth hospitals/HSAs, two assumptions were made due to lack of the information on patients' hospital selection: (1) patients tended to go to the nearest hospitals and (2) if there was a certain percentage of discharges from a hospital within a given ZIP code, those patients should most likely live in the nearest BGs (to the hospital) which contained the same percentage of the population over that ZIP code. The distance was measured along the shortest path on road networks from the population-weighted centroids of BGs (Appendix 2) to each hospital or to the population-weighted centroid of each HSA (Appendix 3). The BGs in each ZIP code were ranked by distance to each hospital/HSA.

In each ZIP code, starting from the first hospital/ HSA, each hospital (i.e., BG in which the hospital was located) or HSA continued to merge with the nearest BG until the summed population proportion of the merged BGs reached the percentage of discharges from that hospital/HSA. As the sum of five (or less) highest percentages of discharges may be less than $100 \%$ within a ZIP code (i.e., some discharges were from other hospitals than these numbered hospitals/HSAs), the unassigned BGs in each ZIP code were allocated by default to its first hospital/HSA. Each cluster of BGs assigned to the same hospital/HSA formed a combined HSA. A visual examination was undertaken to enforce the geographic contiguity of all ZIP codes and BGs within each combined HSA (discontinuity by natural water bodies was allowed). The final output was named the censusderived HSAs (Fig. 2).

\section{Ratio of HBtP}

The population in all ZIP codes and BGs within each HSA was summed to yield the population in that HSA. Then, the HBtP ratio was calculated for each HSA by dividing the total number of hospital beds by the number of the population within that HSA. Local Moran's I (Anselin et al. 2006) was used to identify spatial clusters of HSAs with ratios similar in magnitude (i.e., clusters of high or low ratios) and spatial outliers (i.e., high ratios surrounded by low ratios, or conversely, low ratios surrounded by high ratios) across Florida, based on HSA locations and ratios simultaneously. Any adjacent spatial unit could have ecological, social, and health impacts on the target unit; hence, the first order rook contiguity was applied, which means that all HSAs sharing at least a point-length border with the target HSA are defined as neighbors (Jia et al. 2016). Additionally, the HBtP ratios were also calculated within counties with the clustering patterns examined based on the same contiguity rule. 
Fig. 1 A flowchart of assigning hospitals to ZIP codes

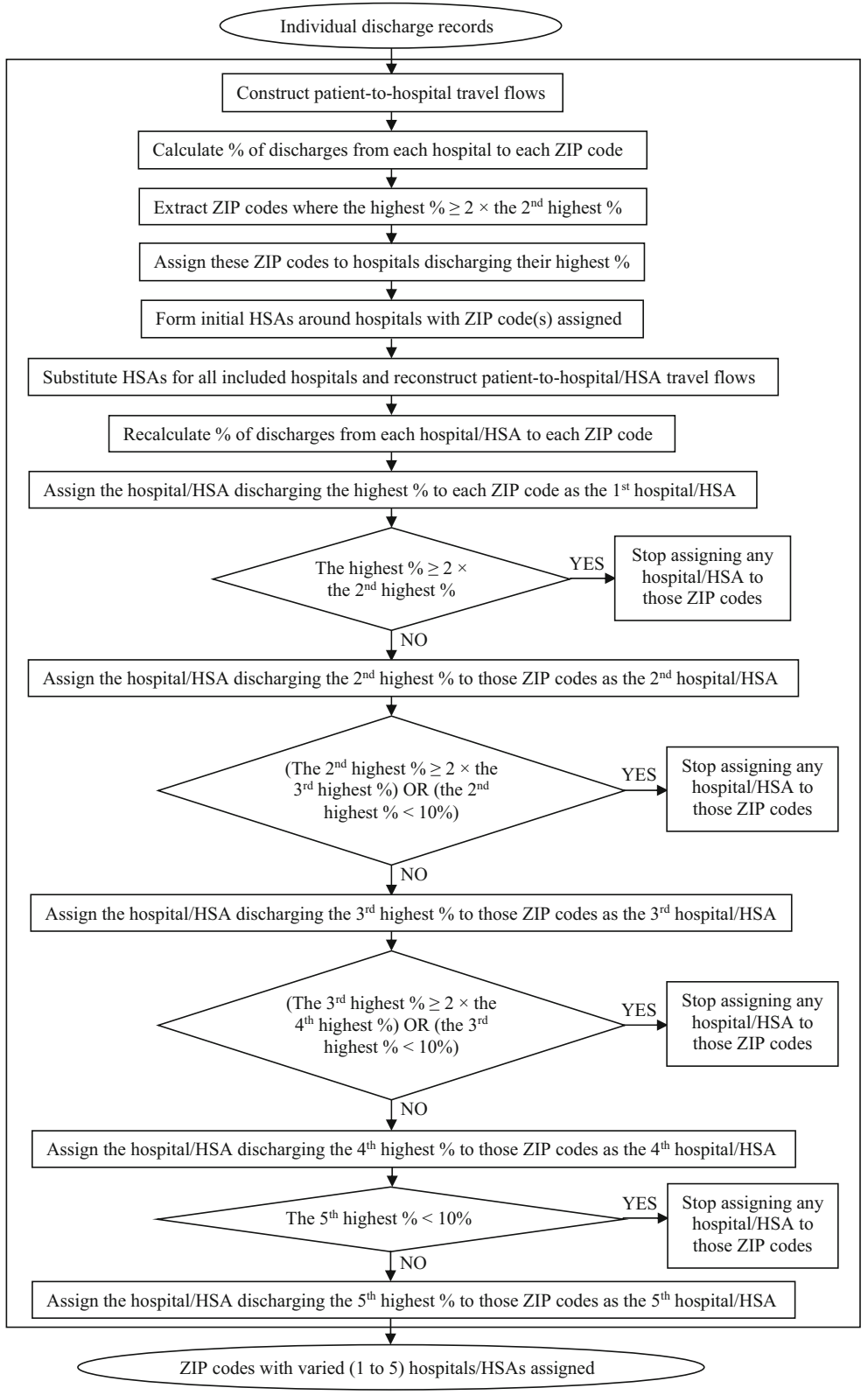

\section{Comparison between HSAs}

The HSA products are usually validated by measuring the degree of self-containment of HSAs, i.e., what percentage of patients within an HSA actually visit local hospital(s) within that HSA. For such purpose, a localization index (LI) is defined as the fraction of discharges of residents that occur within an HSA over all discharges living in that HSA (Klauss et al. 2005). To compare the census-derived HSAs with other HSA products, as done in previous studies (Jia et al. 2015;
Klauss et al. 2005), a rule of $L I \geq 0.5$ was applied to forcing each HSA with $\mathrm{LI}<0.5$ to be merged into an adjacent HSA, which either geographically encircled that HSA or discharged the second highest percentage of patients to that HSA. Merging and testing were iteratively conducted until the LIs of all HSAs $\geq 0.5$.

A $t$ test was used to assess whether there were significant differences between LIs of the aggregated census-derived HSAs and the Dartmouth-derived HSAs. A natural log transformation was conducted to alleviate the skewed distribution of two groups of LIs. 


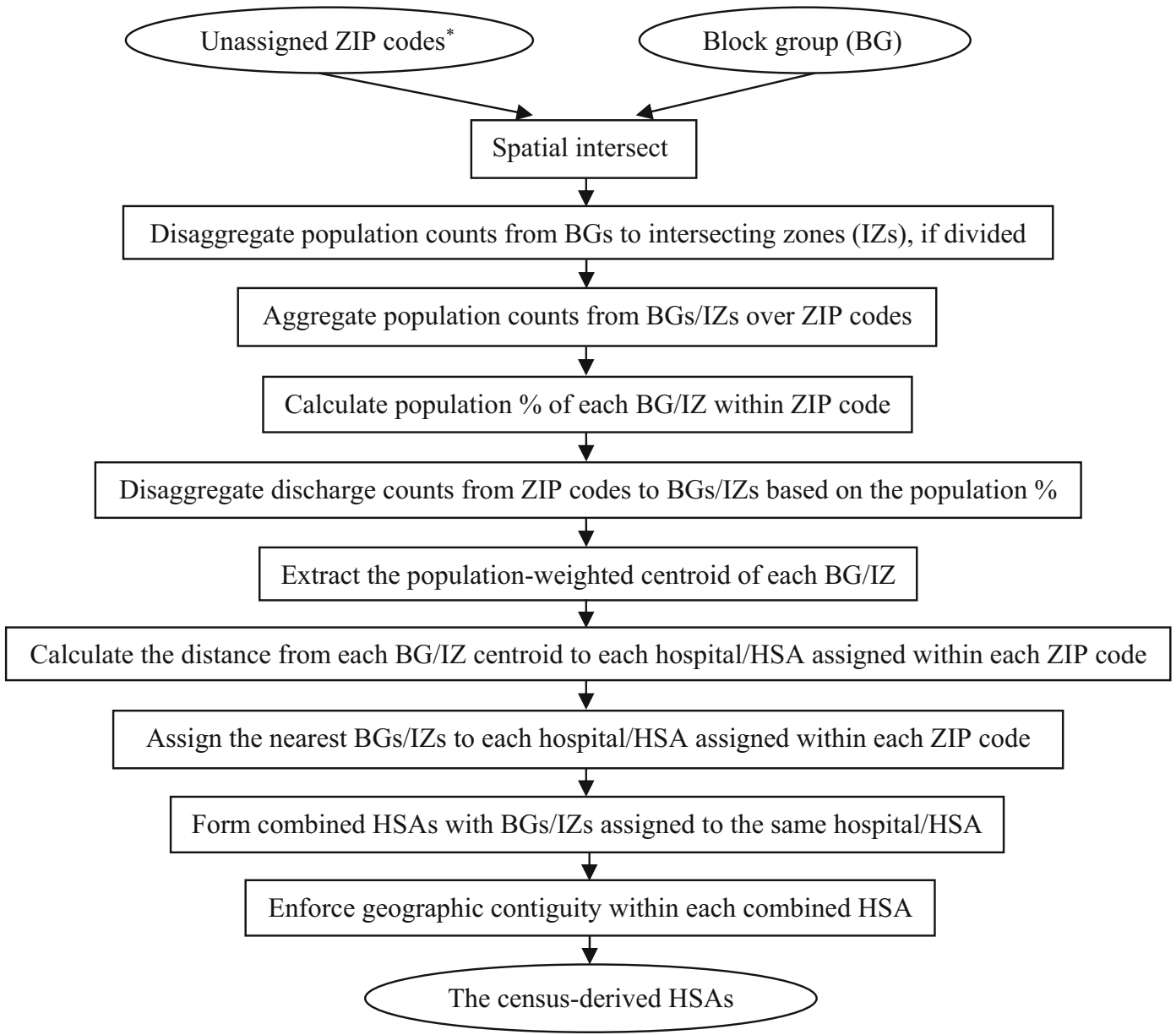

Fig. 2 A flowchart of generating the census-derived hospital service areas (HSAs). Unassigned ZIP codes are the output of Fig. 1, i.e., those ZIP codes which were not assigned to hospitals as a whole, with varied (one to five) hospitals/HSAs assigned (asterisk)

All statistical analyses were performed using SPSS version 24.0 (IBM Corp., Armonk, NY). Implementation of all spatial analyses was conducted in ArcGIS (Version 10.4.1, ESRI, Redlands, CA).

\section{Results}

\section{Census-derived HSAs}

The 221 general hospitals were located in 58 out of 67 counties in Florida, where the HBtP ratios (beds per 1000 people) ranged from 0.4 (Suwannee County) to 5.1 (Alachua County) (Fig. 3). Three counties (Hendry, Levy, and Nassau counties) with low ratios of HBtP were found to be surrounded by counties with significantly higher ratios, while Alachua County had a high ratio and was surrounded by counties with significantly lower ratios. There were 217,243 $(983 \times 221)$ patient-tohospital flows naturally formed from 983 ZIP codes to 221 hospitals in Florida, of which 37,216 flows contained at least one hospital visit, according to the actual discharge records in the year 2011. Nearly half the ZIP codes $(47.3 \%, 465 / 983)$ had the percentage of discharges from the first hospital equal to or greater than two times the percentage of discharges from the second hospital, which thus were assigned to their first hospitals. The unassigned 518 ZIP codes were divided into BGs and assigned to different initial HSAs.

A total of 166 units were produced in the censusderived HSAs, with $80 \%$ of units including only one hospital, $13 \%$ including two, and $7 \%$ including three or more. Compared to the HBtP ratios at the county level, larger spatial heterogeneities in the ratios were observed 


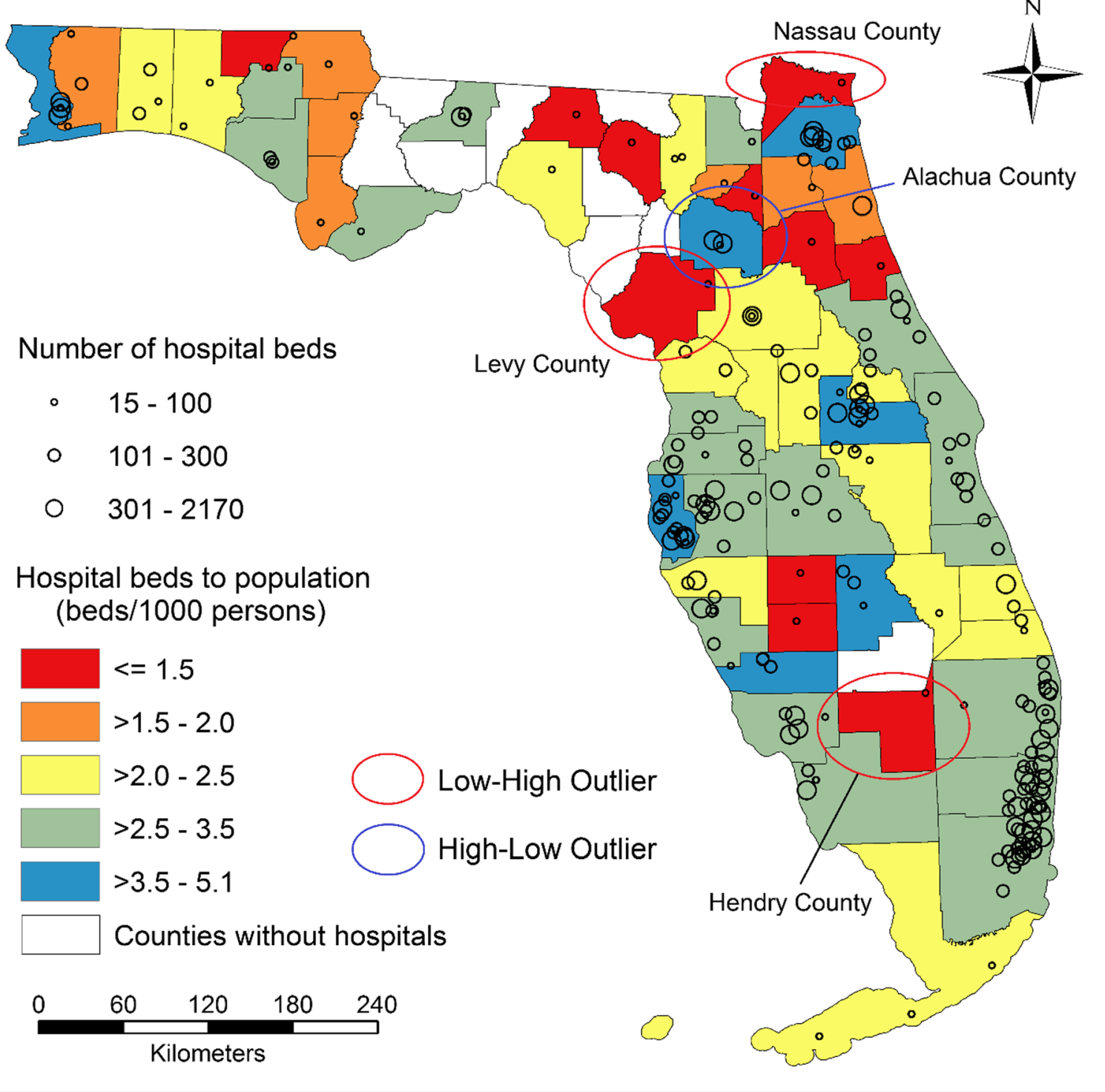

Fig. 3 The ratios of hospital beds to population within counties and the distribution of hospitals with the numbers of hospital beds represented by the size of circles

across HSAs (Fig. 4), where the difference between the most over- and under-served areas was found to be approximately 60 times, i.e., 36.3 in Largo (to the south of Clearwater) versus 0.6 in Safety Harbor (to the east of Clearwater). Seventy-eight percent (130/166) of the HSAs showed ratios between one and five. A weak correlation (Pearson's $r=0.24$ ) was found between the HBtP ratios and the population density at HSA level (Fig. 5). The smaller-sized HSAs in metropolitan areas tended to have higher ratios. For example, a small significant cluster of high ratios was detected in Miami metropolitan area, while a cluster of low ratios was found in Jacksonville metropolitan area.

Aggregation of census-derived HSAs

The 166 units in census-derived HSAs were combined into 78 units in aggregated census-derived HSAs after enforcing $L I \geq 0.5$, which happened to be the same number of units in the Dartmouth-derived HSAs (Fig. 6). The boundaries of the aggregated censusderived HSAs were not aligned with the boundaries of 

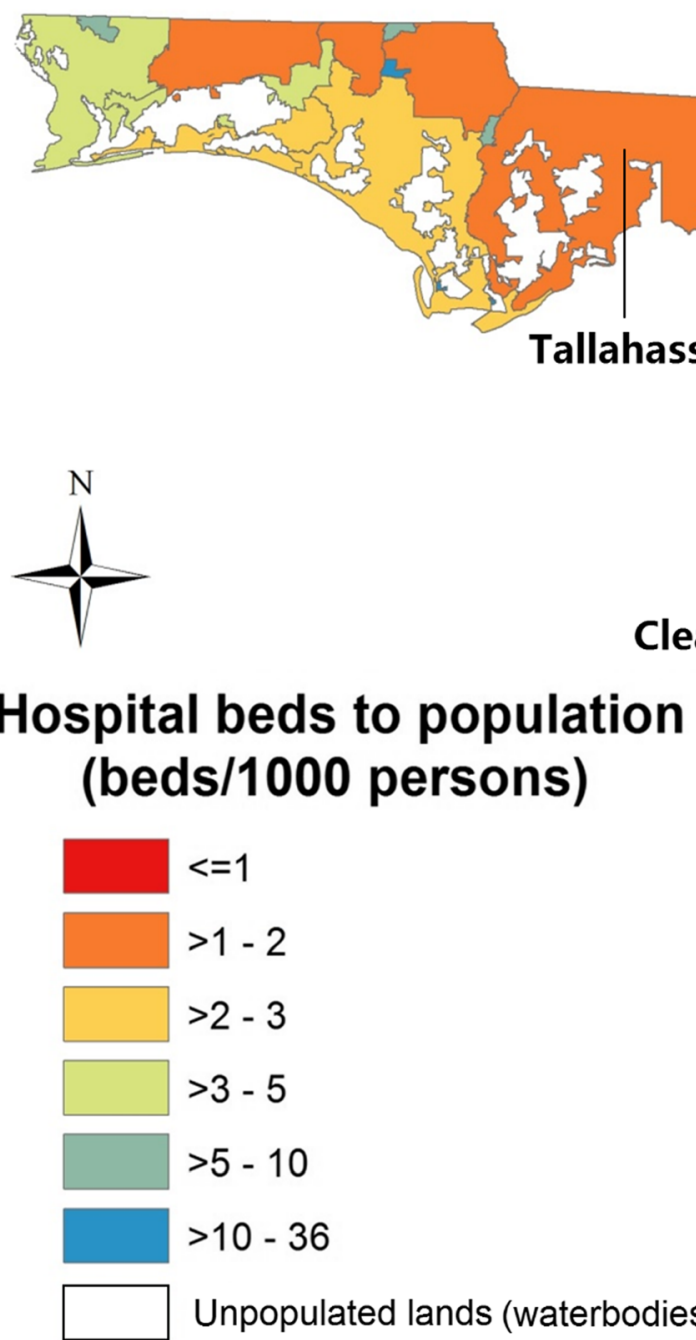

$<=1$

$>1-2$

$>2-3$

$>3-5$

$>5-10$

$>10-36$

Unpopulated lands (waterbodies, wildlife areas, etc.)

0

60

120

180

240 Kilometers

Tampa

Jacksonville

e

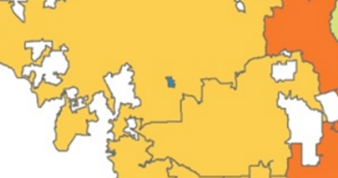

(3)

Orlando

Clearwater 


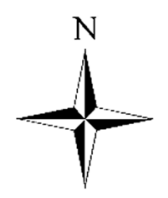

Population density (per square $\mathrm{km}$ )
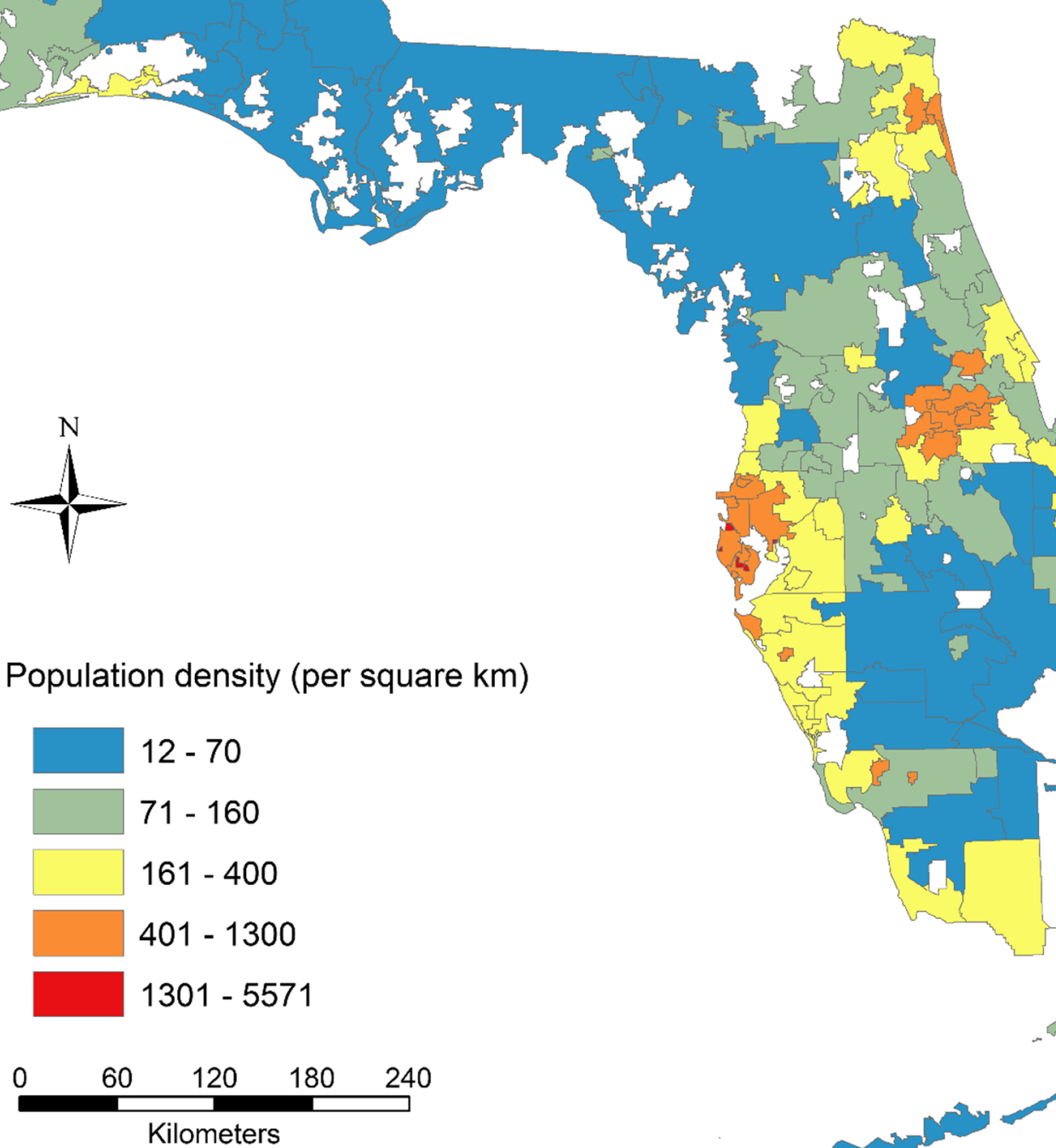

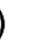




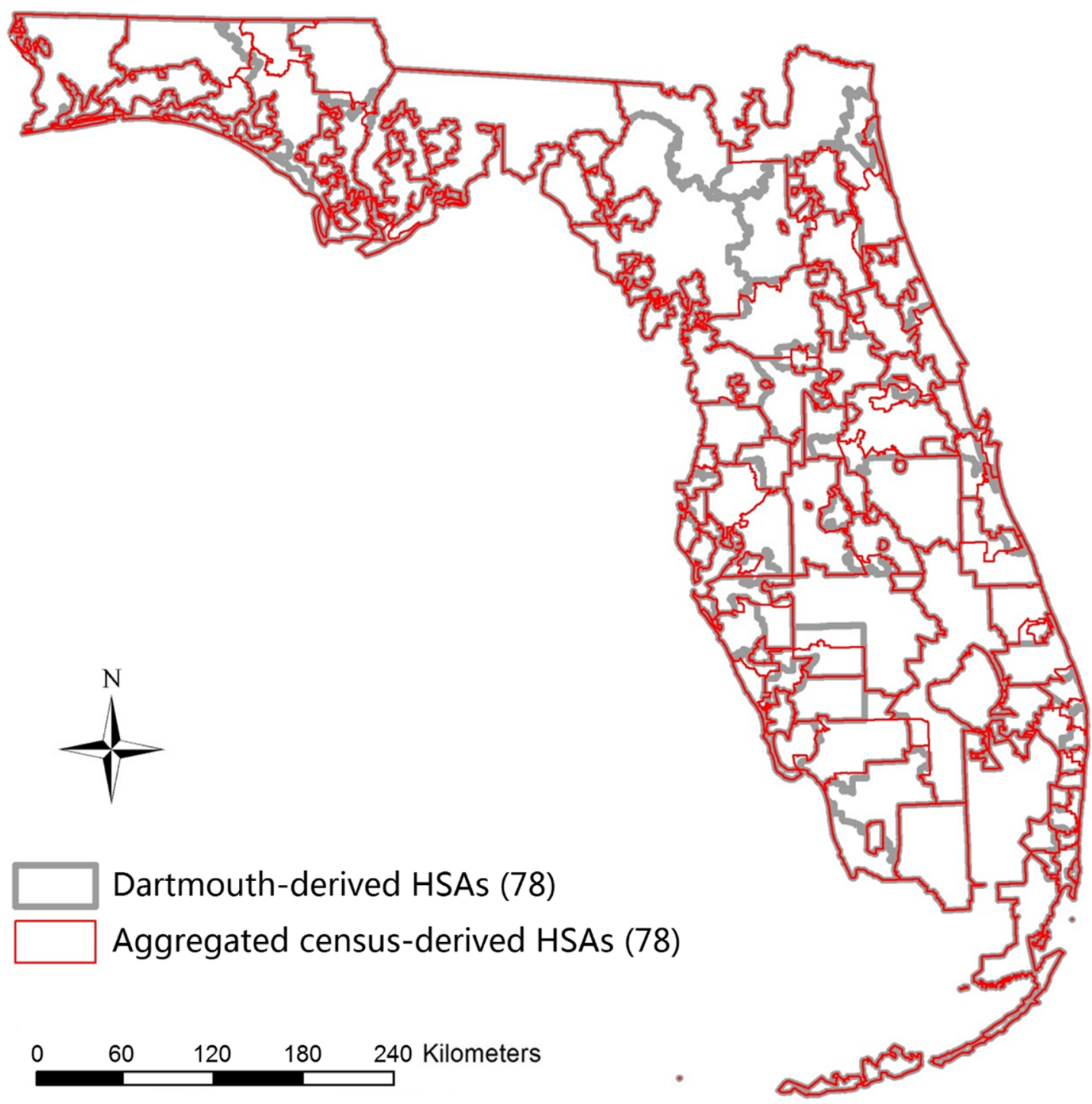

Fig. 6 Boundaries of the Dartmouth-derived HSAs and aggregated census-derived HSAs

available through the American Community Survey in 2005, they were still derived from Census population counts and thus remained limited in temporal resolution and accuracy (especially in less populated areas). Given an inherent relationship between patients and the population, the increasingly available patient data annually in the USA (e.g., SID, State Ambulatory Surgery and Services Databases, State Emergency Department Databases) could be potentially utilized to pursue the annual population estimates with higher accuracy.
The skeleton of this delineation method consists of iterative spatial intersection, computation, neighbor searching, and network analysis, which have been greatly supported and facilitated by GIS and could be easily automated. The census data and GIS have been used together to advance many aspects of population and health research (Jia et al. 2014, 2016; Jia and Gaughan 2016; Luo and Wang 2003); however, no efforts have been made to delineate or improve the HSAs for specific purposes, such as monitoring the equity of hospital 


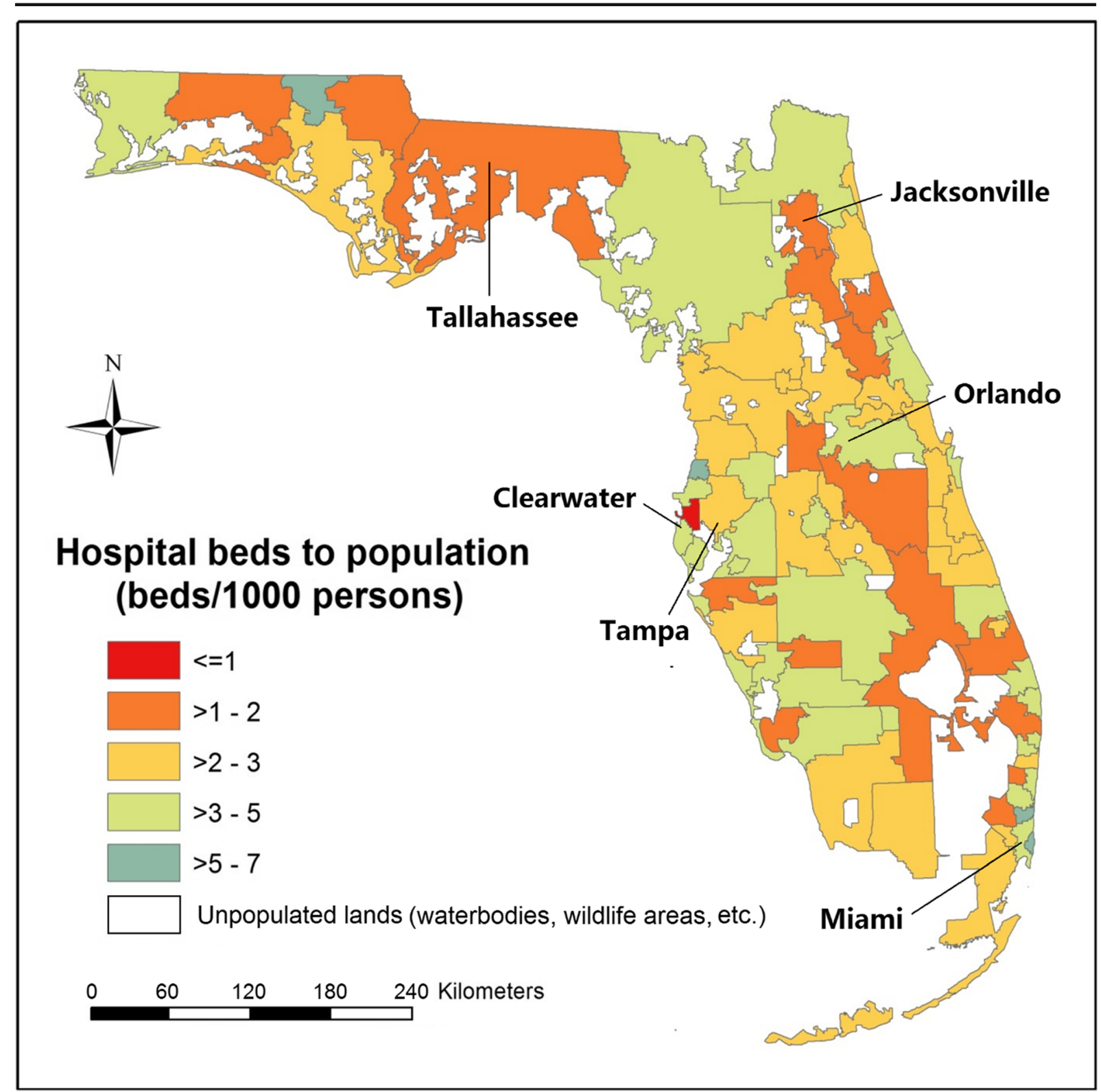

Fig. 7 The ratios of hospital beds to population within the aggregated census-derived HSAs

resources between supply and demand (a surplus of beds, if not considering operation costs and physician and nurse workforce, could cause an overutilization of hospital services as stated in Roemer's Law (Shain and Roemer 1959)).

This method has two major advantages over the previous approaches of HSA delineation (Center for Evaluative Clinical Sciences 1999; Jia et al. 2015, 2017a, b; Jia 2016; Klauss et al. 2005). First, previous approaches produce HSAs that usually contain multiple hospitals in one unit, which creates a higher level of difficulty in assigning responsibility for variation to individual hospitals due to lack of the HSA managers who can coordinate all hospitals within an HSA and manage the HSA as a whole. The census-derived approach seeks to form as many separate HSAs with as few hospitals within each HSA as possible, where each hospital could have its own HSA as long as it serves the highest percentage of patients in its ZIP code. This greatly reduces unnecessary complexities when focus is on the population-related demand for healthcare resources (e.g., hospital beds, physicians), which may 
interest relevant stakeholders and contribute to planning and optimization of hospital resource allocation and healthcare policy-making.

Second, previous approaches fail to consider unrecorded flows and potential demand within HSAs (Brown and Hincks 2008). More often than not, besides the largest percentage of discharges from a certain hospital to a given ZIP code, the percentage of the remaining discharges in that ZIP code from other hospitals may still be considerable. For example, imagine a ZIP code where $51 \%$ of discharges are from one hospital and $49 \%$ are from other(s). Previous approaches only consider the largest percentage and simply assign all discharges in that ZIP code to the hospital which discharges only $51 \%$ of patients, which would result in the biased estimation of patient numbers within HSAs. Such bias may propagate its ways into evaluation of local hospital resource allocation as multiple hospitals in one HSA could complicate assigning the responsibility to each hospital, resulting in inappropriate intervention and policy making. The census-derived approach enables BGs, used as ancillary data, to split up that ZIP code into multiple intersected zones and assigns each intersected zone to the nearest hospital (or adjacent HSA). Therefore, boundaries of the census-derived HSAs should be less subject to fluctuations over time than of HSAs based on patient travel flows, which might present only a transient reflection of unknown demand at a certain point in time.

Although a value of $50 \%$ has been used as a minimum cutoff percentage (of discharges) in previous delineation methods for assigning a ZIP code to a hospital (Jia et al. 2015; Jia 2016; Klauss et al. 2005), a one-sizefits-all cutoff value remains debatable. In this study, whether the largest percentage was twice the second largest percentage of discharges from a different hospital determined whether a ZIP code would be assigned to the hospital discharging the largest percentage of its patients. Such a relative and unconstrained cutoff percentage could further prevent bias caused by assigning a ZIP code with comparative percentages of discharges from different hospitals to only one hospital. This provides more flexibility at different locations than an absolute and one-size-fits-all percentage.

There are some limitations in this study. First, the population and discharges might not be homogeneously distributed within ZIP codes. Thus, there exists uncertainty in the estimated numbers of discharges within BGs. The correlation between the numbers of hospital discharges and the population also remains in doubt at other geographic levels and in other regions, which needs to be verified in more studies. Another major source of uncertainties is the subjective thresholds assigned when deciding whether a ZIP code should be assigned to one or multiple hospitals (i.e., 200\%) and whether assigning should end (i.e., 10\%). Second, in addition to the population number, other demographic information such as age and gender may affect the demand for healthcare supply and should be considered in future efforts. For example, elderly people are usually hospitalized more often and multiple times than adolescents. Also, the high numbers of "snowbird" population (temporary residents), veterans population, and both US and international tourists in Florida should be considered in future studies. Third, 13 unidentified hospitals and 47 psychiatric, rehabilitation, children's, women's, and other specialty hospitals (including about $5.1 \%$ of all discharging records) were excluded from this study, which might affect results. Besides the number of hospital beds, more attention should be paid to the type and range of health services provided by hospitals, as well as outpatient care. Fourth, the patterns of HBtP ratios in this study were different from the patterns among counties or other HSA boundaries. This is also commonly known as the modifiable areal unit problem (MAUP) that conclusions drawn at one geographic level may change when a different analysis unit is used (Openshaw 1984). The relationships among these patterns at different scales need to be discussed further in future studies. Additionally, patients do not always visit the nearest hospitals, which could be affected by the competition among hospitals and other underlying factors (e.g., health insurance, hospital rank/reputation, quality of care). Thus, there exists uncertainty of patients' decision making in hospital selection within ZIP codes, which may be tested by questionnaire survey at the local scale. More in-depth analyses are also warranted to examine the factors that might affect the hospital selection by patients. Moreover, HSA boundaries could inform which hospitals nearby might have impacts on residents at any location, and hence, spatial accessibility of hospital resources could be explored using floating catchment area metrics at finer scales.

\section{Conclusions}

Patients' addresses within ZIP codes will remain unavailable due to confidentiality protection that may last 
for an unexpectedly long period. On the basis of multidisciplinary principles and both actual hospital data and potential population data, this study expands the science of HSA delineation by demonstrating an application of GIS in healthcare planning. The resulting censusderived HSAs allow policy-makers to easily identify the geographical disparity in per capita share of hospital resources and assign responsibility to specific hospitals and areas, providing an appropriate spatial scale for evaluating and improving current healthcare planning and delivery. The method is particularly applicable for public health professionals and health services researchers. What is more, the strong correlation between numbers of hospital discharges and the population within ZIP codes makes each a good indicator for the other. This discovery holds a remarkable potential for affordable population estimation, especially in non-census years, and deserves to be substantially explored by incorporating other supplementary data sources (e.g., outpatient visit data).

Acknowledgments Peng Jia, Director of the International Initiative on Spatial Lifecourse Epidemiology (ISLE), thanks Lorentz Center, the Netherlands Organization for Scientific Research, the Royal Netherlands Academy of Arts and Sciences, the Chinese Center for Disease Control and Prevention, and the West China School of Public Health in Sichuan University for funding the ISLE and supporting ISLE's research activities. We would also like to thank three anonymous reviewers for very helpful comments.

Funding information This study was partly supported by the International Strategic Partnerships (Grant No. CvB-UIT$3513 \backslash \mathrm{S} \& B$ ) and the China Medical Board (Grant No. 16-262)

Open Access This article is distributed under the terms of the Creative Commons Attribution 4.0 International License (http:// creativecommons.org/licenses/by/4.0/), which permits unrestricted use, distribution, and reproduction in any medium, provided you give appropriate credit to the original author(s) and the source, provide a link to the Creative Commons license, and indicate if changes were made.

\section{References}

Agency for Healthcare Research and Quality. (2011). Healthcare cost and utilization project (hcup) state inpatient databases (sid)-florida. Rockville, MD.

Anderson, G. F., Hussey, P. S., Frogner, B. K., \& Waters, H. R. (2005). Health spending in the United States and the rest of the industrialized world. Health Affairs, 24, 903-914.
Anselin, L., Syabri, I., \& Kho, Y. (2006). Geoda: an introduction to spatial data analysis. Geographical Analysis, 38, 5-22.

Ashton, C. M., Petersen, N. J., Souchek, J., Menke, T. J., Yu, H. J., Pietz, K., Eigenbrodt, M. L., Barbour, G., Kizer, K. W., \& Wray, N. P. (1999). Geographic variations in utilization rates in veterans affairs hospitals and clinics. The New England Journal of Medicine, 340, 32-39.

Brown, P. J., \& Hincks, S. (2008). A framework for housing market area delineation: principles and application. Urban Studies, 45, 2225-2247.

Center for Evaluative Clinical Sciences. (1999). The Dartmouth atlas of health care in the United States. Chicago, Illinois.

Claval, P. (1987). The region as a geographical, economic and cultural concept. International Social Science Journal, 39, 159-172.

Fisher, E. S., Wennberg, J. E., Stukel, T. A., \& Sharp, S. M. (1994). Hospital readmission rates for cohorts of Medicare beneficiaries in Boston and new haven. The New England Journal of Medicine, 331, 989-995.

Goodchild, M.F., \& Lam, N.S.N. (1980). Areal interpolation: a variant of the traditional spatial problem. London, Ont.: Department of Geography, University of Western Ontario.

Hogan, C. (1988). Patterns of travel for rural individuals hospitalized in New York state: relationships between distance, destination, and case mix. The Journal of Rural Health, 4, 29-41.

Horev, T., Pesis-Katz, I., \& Mukamel, D. B. (2004). Trends in geographic disparities in allocation of health care resources in the us. Health Policy, 68, 223-232.

Jackson, C.A., Derose, K.P., Chiesa, J., Escarce, J.J., Chicago. (2002). Hospital care for the uninsured in Miami-dade county. Hospital finance and patient travel patterns. Santa Monica, CA:RAND CORP.

Jia, P. (2016). Developing a flow-based spatial algorithm to delineate hospital service areas. Applied Geography, 75, 137-143.

Jia, P., \& Gaughan, A. E. (2016). Dasymetric modeling: a hybrid approach using land cover and tax parcel data for mapping population in Alachua county, Florida. Applied Geography, 66, 100-108.

Jia, P., \& Xierali, I. (2015). Disparities in patterns of health care travel among inpatients diagnosed with congestive heart failure, Florida, 2011. Preventing Chronic Disease, 12, E150.

Jia, P., Qiu, Y., \& Gaughan, A. E. (2014). A fine-scale spatial population distribution on the high-resolution gridded population surface and application in Alachua county, Florida. Applied Geography, 50, 99-107.

Jia, P., Xierali, I., \& Wang, F. (2015). Evaluating and redemarcating the hospital service areas in Florida. Applied Geography, 60, 248-253.

Jia, P., Anderson, J. D., Leitner, M., \& Rheingans, R. (2016). High-resolution spatial distribution and estimation of access to improved sanitation in Kenya. PLoS One, 11, e0158490.

Jia, P., Wang, F., \& Xierali, I. (2017a). Delineating hierarchical hospital service areas in Florida. Geographical Review, 107, 608-623.

Jia, P., Wang, F., \& Xierali, I. (2017b). Using a huff-based model to delineate hospital service areas. The Professional Geographer, 69, 522-530.

Jones, A. P., Haynes, R., Sauerzapf, V., Crawford, S. M., Zhao, H., \& Forman, D. (2008). Travel time to hospital and treatment for breast, colon, rectum, lung, ovary and prostate cancer. European Journal of Cancer, 44, 992-999. 
Klauss, G., Staub, L., Widmer, M., \& Busato, A. (2005). Hospital service areas - a new tool for health care planning in Switzerland. BMC Health Services Research, 5, 33.

Krueger, P. M., Bhaloo, T., \& Rosenau, P. V. (2009). Health lifestyles in the U.S. and Canada: are we really so different? Social Science Quarterly, 90, 1380-1402.

Luo, W., \& Wang, F. (2003). Measures of spatial accessibility to health care in a GIS environment: synthesis and a case study in the Chicago region. Environment and Planning B, 30, 865-884.

Mansfield, C. J., Wilson, J. L., Kobrinski, E. J., \& Mitchell, J. (1999). Premature mortality in the United States: the roles of geographic area, socioeconomic status, household type, and availability of medical care. American Journal of Public Health, 89, 893-898.

Miller, P. (1994). Medical center uses desktop mapping to cut costs and improve efficiency. Geographic Information Systems, 4, 40-41.

Moist, L. M., Bragg-Gresham, J. L., Pisoni, R. L., Saran, R., Akiba, T., Jacobson, S. H., Fukuhara, S., Mapes, D. L., Rayner, H. C., Saito, A., \& Port, F. K. (2008). Travel time to dialysis as a predictor of health-related quality of life, adherence, and mortality: the dialysis outcomes and practice patterns study (dopps). American Journal of Kidney Diseases, 51, 641-650.

Openshaw, S. (1984). The modifiable areal unit problem. Norwich: Geo Books.

Patel, A. R., Fik, T. J., \& Thrall, G. I. (2008). Direction sensitive wedge-casting for trade area delineation. Journal of Real Estate Portfolio Management, 14, 125-140.
Rodwin, V. G., \& Sandier, S. (1993). Health care under French national health insurance. Health Affairs, 12, 111-131.

Shain, M., \& Roemer, M. I. (1959). Hospital costs relate to the supply of beds. Journal of Occupational and Environmental Medicine, 1, 518.

Shortt, N. K., Moore, A., Coombes, M., \& Wymer, C. (2005). Defining regions for locality health care planning: a multidimensional approach. Social Science \& Medicine, 60, 27152727.

Smith, C. J. (1998). Modernization and health care in contemporary China. Health \& Place, 4, 125-139.

The World Bank. (2017). World development indicators - hospital beds (per 1,000 people) in the United States.

US Census Bureau. (2014). Tiger/line shapefiles. U.S. Department of Commerce website:U.S. Department of Commerce.

Wennberg, J. E. (1999). Understanding geographic variations in health care delivery. The New England Journal of Medicine, $340,52-53$.

Wilbush, J. (1974). The local hospital: functions, advantages and difficulties. Canadian family physician Medecin de famille canadien, 20, 54-56.

Zhang, Y., Baik, S. H., Fendrick, A. M., \& Baicker, K. (2012). Comparing local and regional variation in health care spending. The New England Journal of Medicine, 367, 1724-1731.

Publisher's note Springer Nature remains neutral with regard to jurisdictional claims in published maps and institutional affiliations. 